\title{
Die Europäische Union
}

\section{Governance und Policy-Making}

Herausgegeben von

Ingeborg Tömmel 
Bibliografische Information Der Deutschen Nationalbibliothek

Die. Deutsche Nationalbibliothek verzeichnet diese Publikation in der

Deutschen Nationalbibliografie; detaillierte bibliografische Daten sind im Internet über

$<$ http://dnb.d-nb.de> abrufbar.

1. Auflage 2008

Alle Rechte worbehalten

(1) VS Verlag für Sozinlwissenschaften I GWV Fachverlage GmbH, Wiesbaden 2008

Lektorat: Frank Schindler

Der VS Verlag für Sozialwissenschaften ist ein Unternehmen von Springer Science+Business Media.. www.s-verlagde

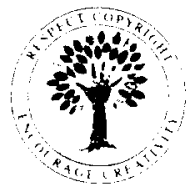

Das Werk einschließlich aller seincr Tcile ist urheberrechelich geschützt. Jede Verwertung aufierhalb der engen Grenzen des Urheberrechtsgesetzes ist ohne Zustimmung des Verlays unzulässig und strafbar. Das gilt insbesondere für Vervieltältigungen, Ühersetzungen, Mikroverfilmungen und die Finspeicherung und Verarbeitung in elektronischen Systemen.

Die Wiedergabe won Gebrauchsnamen, Handelsnamen, Warenbezcichnungen usw. in diesem Werk berechtigt auch ohne besondere Kennzeichnuny nicht zu der Annahme, dass solche Namen im Sinne der Warenzeichen- und Markenschutz-Gesetzgebung als frei zu betrachen wären und daher von jedermann benutzt werden dürten.

Druck und buchbinderische Verarbeitung: Wilhelm \& Adam, Heusenstamm

Satz: ITS Text und Satz Anne Fuchs, Bamberg

Gedruckt auf säurefreiem und chlorfrei gebleichtem Papier

Printed in Germany 


\section{Inhaltsverzeichnis}

Vorwort .......................... 9

\section{Theoretische Perspektiven}

\section{Ingeborg Tömmel}

Governance und Policy-Making im Mehrebenensystem der EU . . . . . . . .

Artbur Benz

Entwicklung von Governance im Mehrebenensystem der EU . . . . . . . . . 36

\section{II. „Harte“ versus „weiche“ Modi der Governance}

Tanja A. Börzel

European Governance - Verhandlungen und Wettbewerb im Schatten der Hierar-

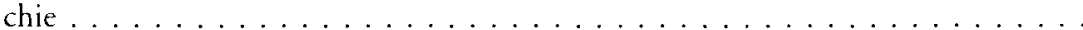

Holger Bähr / Oliver Treib / Gerda Falkner

Von Hierarchie zu Kooperation? Zur Entwicklung von Governance-Formen in zwei regulativen Politikfeldern der EU . . . . . . . . . . . . . . .

Simone Leiber / Armin Schäfer

Der doppelte Voluntarismus in der E.U-Sozial- und Beschäftigungspolitik .

\section{Kompetenzverschiebungen im Mehrebenensystem}

\section{Miriam Hartlapp}

Intra-Kommissionsdynamik im Policy-Making: EU-Politiken angesichts des demographischen Wandels

\section{Andrea Lenschow / Renate Reiter}

"Keeping Competence" - Politikkoordination als Mittel der institutionellen Verankerung europäischer Umwelt- und Regionalpolitik . . . . . . . . . . . .

Michael W. Bauer / Christoph Knill

Politikabbau im europäischen Mehrebenensystem: Nationale Beendigungseffekte europäischer Politik 


\section{Herausbildung regulativer Regime im Mehrebenensystem}

Edgar Grande / Ute Hartenberger

Regulatory Governance im Europäischen Mehrebenensystem . . . . . . . . . . 209

Thomas Gehring / Michael A. Kerler / Sebastian Krapobl

Risikoregulierung im europäischen Binnenmarkt: Regulierungsagenturen, Normungsinstitute und Komitologie-Ausschüsse . . . . . . . . . . . . . . . 23

Shawn Donnelly

Regime der Finanzmarkt- und Unternehmensregulierung in Europa: die Bestimmung gesetzlicher Strukturen und Regulierungsprozesse

\section{Governance im Schatten der Marktintegration}

Susanne K. Schmidt / Michael Blauberger / Wendelmoet van den Nouland Jenseits von Implementierung und Compliance - Die Europäisierung der Mitglied-

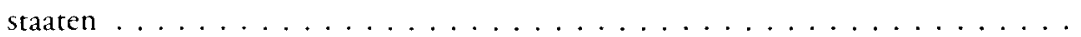

Philipp Genschel / Thomas Rixen / Susanne Uhl

Die Ursachen des europäischen Steuerwettbewerbs

\section{Formen transnationaler Politikkoordination}

Wolfgang Wagner

Europäische Governance im Politikfeld Innere Sicherheit . . . . . . . . . . .

Udo Diedrichs

Neue Dynamik in der Europäischen Außen- und Sicherheitspolitik: auf dem Weg zu einer EU Security Governance

\section{Politikkoordination in internationalen Dimensionen}

Sandra Lavenex / Dirk Lehmkuhl / Nicole Wichmann

Die Nachbarschaftspolitiken der Europäischen Union: zwischen Hegemonie und erweiterter Governance . . . . . . . . . . . . . . . . . . . . . . . . . .

Jürgen Neyer / Ulrike Ehling

Externe Effekte von Mehrebenenregieren. Die Europäische Gemeinschaft in der

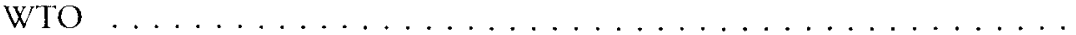




\section{Schlussfolgerungen}

Ingeborg Tömmel

Governance europäischer Politiken - Konvergenzen, Divergenzen und Varianzen im EU-System

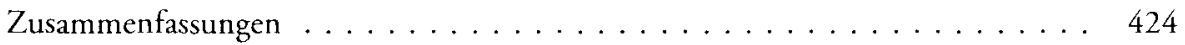

Abstracts ........................... 431

Verzeichnis der Autorinnen und Autoren . . . . . . . . . . . . . . 437 


\title{
Risikoregulierung im europäischen Binnenmarkt: Regulierungsagenturen, Normungsinstitute und Komitologie-Ausschüsse
}

\author{
Thomas Gehring / Michael A. Kerler/Sebastian Krapohl
}

\section{Einleitung}

Die europäische Binnenmarktpolitik wird zunehmend durch Regulierungsregime geprägt, die einen erheblichen Teil der Entscheidungen auf administrative, technische und wissenschaftliche Expertengremien verlagern. Dazu werden neue, mir Entscheidungskompetenzen ausgestattete Foren geschaffen, an denen auch Akteure beteiligr sind, die im europäischen Entscheidungsprozess sonst keine herausragende Rolle spielen (Gehring et al. 2005). Dadurch richtet sich die Funktion der Legislativorgane der EU, also der Kommission, des Rates und des Parlaments, zunehmend auf die Lenkung der nachgeordneten Entscheidungsgremien. Ausgelöst wurde diese Entwicklung durch den „Neuen Ansatz“, der im Rahmen des Binnenmarktprogramms von 1985 (KOM 85 [310]) eingeführt wurde, um die schwierige Entscheidungsfindung im Rat auf dem Gebiet der binnenmarktbezogenen Risikoregulierung teilweise zu umgehen (Joerges 1988). Eingerichtet wurden nacheinander neue Regulierungsregime für die europäische Normung von Produktrisiken, für die Regulierung der Lebensmittelsicherheit, die Arzneimittelzulassung, die Regulierung des europäischen Finanzmarktes sowie die Chemikalienkontrolle. Das Weißbuch der Kommission über Europäisches Regieren (KOM 2001 [428]) sieht weitere Anwendungsbereiche vor.

Die neuen Regulierungsregime steigern die Entscheidungsfähigkeit des politischen Systems der EU auf dem Gebiet der Binnenmarktpolitik erheblich und funktionieren in der Regel vergleichsweise geräuschlos. Bislang ist jedoch nicht geklärt, woher ihr hohes Problemlösungspotential rührt und wo dessen Grenzen liegen. Die Risikoregulierung im Binnenmarkt findet zwar im Schatten der Hierarchie des europäischen Rechts statt, aber die Entscheidungen werden im Rahmen von Verhandlungen getroffen und die jeweils beteiligten Gremien sind zur Kooperation untereinander gezwungen (Benz 2005). Während einige dieser Regulierungsstrukturen weitgehend auf staatlich-administrative Akteure beschräkt sind, spielen nicht-staatliche Akteure in anderen cine hervorgehobene Rolle (Börzel 2005). In einigen Fällen wird verbindliches europäisches Recht geschaffen, während etwa die europäischen technischen Normen kcine rechtiche Verbindlichkeit erlangen. In jedem Fall sind die an der Regulierung beteiligten Netzwerke von Akteuren sehr unterschiedlich zusammengesetzt.

Vorhandene Ansätze in der Governance-Forschung sind entweder zu breit oder zu eng, um einen systematischen Vergleich von supranationalen Regulierungsregimen zu ermöglichen. Auf der einen Seite befasst sich ein Teil der Debatre mit allgemeinen Strukturmerkmalen europäischen Regierens. So wird gemeinhin angenommen, dass die EU sich durch ihr Mehrebenen-Regierungssystem von den politischen Systemen der Mitgliedstaaten unterscheidet (Jachtenfuchs/Kohler-Koch 1996; Marks et al. 1996; 
Scharpf 1997). Demnach findet die Politikgestaltung auf verschiedenen Ebenen statt und gewährt Interessengruppen verstärkte Einflussmöglichkeiten auf den unterschiedlichen Ebenen. Zum anderen wird die europäische Politik zunehmend in Regulierungsnetzwerken gestaltet, in denen öffentliche und private, supranationale und mitgliedstaatliche Akteure sowie Experten und Interessenvertreter zusammenarbeiten (Jachtenfuchs/Kohler-Koch 2004: 77-91; Eberlein/Grande 2005). Die EU ist in hohem Maß auf die Zusammenarbeit in derartigen Netzwerken angewiesen, um supranationale Politik gestalten und anschließend auch umsetzen zu können. Auf der anderen Seite befasst sich ein Teil der Literatur zur Risikoregulierung im Binnenmarkt nur mit einzelnen Regulierungsinstitutionen. So geht Majone (2001) davon aus, eine problemangemessene Risikoregulierung setze voraus, dass Entscheidungen weitgehend unabhängig von politischen Interessen durch Regulierungsagenturen gefällt werden. Im Gegensatz dazu betrachten Joerges und Neyer (1997) das Ausschusswesen als einen EU-spezifischen Regulierungsmodus, der problemadäquate Entscheidungen hervorbringen kann und herkömmliche zwischenstaatliche Verhandlungen in supranationale Deliberation transformiert. Ein Vergleich verschiedener Regime - wie zum Beispiel der Arzneimittelregulierung durch Agenturen, der technischen Normung durch privatrechtliche Organisationen und der Lebensmittelregulierung im Ausschusswesen - ist damit kaum möglich.

In diesem Beitrag untersuchen wir, welche institutionellen Strukturen sich im Bereich der Regulierung von Produktrisiken im europäischen Binnenmarkt entwickelt haben, wie Entscheidungen in ihrem Rahmen getroffen werden und welche Politikergebnisse daraus hervorgehen. Dazu gehen wir in zwei Schritten vor. Zunächst skizzieren wir die Entscheidungsproblematik, die die Regulierung von Produktrisiken im Binnenmarkt aufwirft, und entwickeln eine theoretische Konzeption der Risikoregulierung, die einerseits nicht auf politische Zielbestimmung verzichtet und andererseits hinreichend viel Expertise mobilisiert, um sachgerechte Entscheidungen zu gewährleisten (2). Danach untersuchen wir drei Regulierungsregime, die sich unter jeweils spezifischen Bedingungen in zentralen Bereichen der europäischen Binnenmarktpolitik etablier haben: die EU-weite Zulassung von Arzneimitteln unter Beteiligung einer europäischen Regulierungsagentur, die Normung technischer Güter im Binnenmarkt unter starker Beteiligung nicht-staatlicher Akteure sowie die Regulierung der Lebensmittelsicherheit mittels "traditioneller" Komitologie-Ausschüsse (3).

Wir kommen zu dem Schluss, dass funktional differenzierte und verrechtichte Regulierungsregime erfolgreiche Risikoregulierung im Binnenmarkt ermöglichen. Dies gelingt, indem sie politische Zielbestimmung und auf Expertenwissen gestützte Umsetzungsentscheidungen systematisch miteinander verbinden. In weniger differenzierten und verrechtlichten Regimen gelingt dies hingegen nicht oder nur unvollständig. 


\section{Durch funktionale Differenzierung und Verrechtlichung zur problemadäquaten Risikoregulierung}

\subsection{Die Problematik der Risikoregulierung im europäischen Binnenmarkt}

Die Regulierung von Produktrisiken im Rahmen der europäischen Binnenmarktpolitik liegt an der Schnittstelle von Marktschaffung und Marktkorrektur. Jede Form der Binnenmarktpolitik ist im Kern auf die Beseitigung von Handelshemmnissen gerichter. In vielen Fällen werden die Mitgliedstaaten durch die Entscheidungstätigkeit des Europäischen Gerichtshofes (EuGH) auf die gegenseitige Anerkennung nationaler Schutzstandards verpflichtet (negative Integration, Scharpf 2003). Damit ist jedoch die Gefahr verbunden, dass sich der jeweils niedrigste nationale Standard binnenmarktweit durchsetzt ("race to the bottom"). Deshalb dürfen die Mitgliedstaaten Belange des Umwelt-, Arbeits- oder Verbraucherschutzes im Wege der nationalen Rechtsetzung sicherstellen. In diesen Fällen kann die Marktintegration nur durch das Setzen harmonisierter Schutzstandards erfolgen (positive Integration). Jede Harmonisierung nationaler Maßnahmen zur Regulierung von Produktrisiken hat deshalb sowohl eine binnenmarktpolitische (marktschaffende) als auch eine umwelt-, arbeits- oder verbraucherschutzpolitische (marktkorrigierende) Dimension. Durch einen auf europäischer Ebene harmonisierten Produktstandard verlieren die Mitgliedstaaten die Befugnis, entgegenstehende nationale Schutzgesetzgebung zu erlassen oder aufrechtzuerhalten. Deshalb muss der europäische Standard gewährleisten, dass die Verbraucher und Nutzer der betreffenden Produkte - also etwa Kinder, die mit importiertem Spielzeug spielen, Arbeitskräfte, die gefährliche Maschinen bedienen, Patienten, die mit hochwirksamen, aber auch riskanten Arzneimitteln behandelt werden, oder Konsumenten von Lebensmitteln - hinreichend vor Risiken geschützt werden.

Eine problemadäquate Risikoregulierung muss ein angemessenes Schutzniveau gewährleisten, ohne Wirtschaftsaktivitäten durch überhöhte Anforderungen zu erdrosseln. Auf den Märkten für risikobehaftete Waren herrscht typischerweise eine hochgradige Informationsasymmetrie. Insbesondere die Verbraucher verfügen nicht über alle relevanten Information oder über den Sachverstand, um diese in rationale Kaufentscheidungen umzusetzen (z. B. Feick 2000). Obwohl Konsumenten und Arbeitskräften typischerweise an einem besonders hohen Schutzniveau gelegen ist, müssen sie auch daran interessiert sein, dass Produkte erschwinglich und verfügbar bleiben. Umgekehrt werden Erzeuger tendenziell niedrige Standards befürworten, deren Einhaltung geringere Kosten verursacht, aber im Interesse einer stabilen Marktentwicklung muss ihnen auch an der zuverlässigen Vermeidung von Skandalen gelegen sein. Auf lange Sicht kann man also eine Konvergenz der Interessen unterschiedlicher Interessengruppen erwarten.

Die binnenmarktinduzierte Regulierung von Produktrisiken wirft eine mehrschichtige Entscheidungsproblemarik auf, die dazu führen kann, dass Entscheidungen blockiert oder nach Verteilungsgesichtspunkten gefällt werden. Erstens befinden sich die Mitgliedstaaten vielfach in einer widersprüchlichen Interessenlage, die die Kooperationsproblematik des Gefangenendilemmas aufwirft. Sie haben ein Interesse an einem europäischen Binnenmarkt, in dem der Wohlfahrt schaffende Warenaustausch nicht durch national unterschiedliche Produktanforderungen gestört wird, können aber im Einzelfall durchaus geneigt sein, nicht konkurrenzfähige nationale Produktionsbereiche 
zu schürzen oder Arbeitsschutz und Volksgesundheit in ihrem jeweiligen Hoheitsgebiet durch nationale Rechtsetzung sicherzustellen. Wenn kurzfristige und langfristige Interessen auseinanderlaufen, können Akteure sich nicht mehr konsistent verhalten (Elster 1979: 67). Zueitens befinden sich die Mitgliedstaaten untereinander im Rahmen der Ratsverhandlungen, die das zentrale Nadelöhr der europäischen Rechtsetzung bilden, zusätzlich in einer schwierigen Koordinationssituation mit Verteilungskonflikt. Vielfach existieren in den nationalen Verwaltungstraditionen (Héritier et al. 1994) unterschiedliche Regulierungsansätze, oder einige Staaten präferieren grundsätzlich ein vergleichsweise niedriges, andere dagegen ein hohes Schutzniveau. Dann droht dem zwischenstaatlichen Entscheidungsprozess die Blockade. Unter diesen Bedingungen werden die getroffenen Regulierungsentscheidungen sich weitgehend an ihren Verteilungsfolgen und der jeweils vorherrschenden Machtverteilung orientieren, sodass problemadäquate Ergebnisse kaum zu erwarten sind.

Zur Entschärfung dieser Entscheidungsproblematik könnten unabhängige Agenturen eingerichtet werden (Majone 2001), die jedoch neue Probleme aufwerfen. Die Übertragung der Regulierungsentscheidungen auf eine unabhängige Agentur ist darauf gerichtet, die politischen Akteure institutionell so zu binden, dass sie nicht mehr in die Einzelfallentscheidungen eingreifen können. Ein solches, aus der Währungspolitik bekanntes Arrangement soll angesichts zeitlich inkonsistenter Präferenzen eine „glaubwürdige Bindung" (Moravcsik 1998: 73-77) der politischen Akteure an ihre übergreifenden Ziele gewährleisten. Allerdings können die beauftragten Agenturen eigene Interessen verfolgen oder von dritten Interessen beeinflusst werden (Moe 1990). Der hier untersuchte Bereich der Risikoregulierung wirft insbesondere das Problem der gezielten Beeinflussung durch gut organisierte Interessengruppen (regulatory capture) auf. So könnte es einer vergleichsweise kleinen Gruppe von Herstellern riskanter Produkte gelingen, Regulierungsentscheidungen in ihrem Sinn zu beeinflussen. Der „PrinzipalAgenten"-Ansatz betont daher, dass die Übertragung von Entscheidungskompetenzen auf nachgeordnete Akteure immer mit Kontrollverlusten und dem Risiko unerwünschter Entscheidungen einhergeht (Pollack 2003). Insgesamt wirft die Übertragung weitreichender Regulierungskompetenzen auf unabhängige Agenturen erhebliche Probleme auf. Sie hat sich in der Praxis auch deshalb als wenig akzeptabel für die Mitgliedstaaten erwiesen, weil sie das in der EU etablierte „institutionelle Gleichgewicht“ stören würde (Majone 2002).

\subsection{Problemadäquate Regulierung durch funktionale Differenzierung und Verregelung des Entscheidungsprozesses}

In funkrional differenzierten Entscheidungssystemen können zwei Teilfunktionen der Regulierung von Produktrisiken im europäischen Binnenmarkt, nämlich die allgemeine Zielbestimmung und die Detailumsetzung, getrennt voneinander ausgeführt werden. Auf der einen Seite müssen grundsätzliche Zielbestimmungen etwa darüber vorgenommen werden, in welchem Ausmaß Verbraucher oder Anwender eines inhärent gefährlichen Gutes vor Risiken geschützt werden, beziehungsweise welchen allgemeinen Grundsätzen die Zulassung oder Beschränkung der Marktfähigkeit derartiger Güter unterliegen sollen. Auf der anderen Seite müssen Detailentscheidungen darüber getroffen wer- 
den, welche Folgen sich daraus für das konkrete Produkt beziehungsweise für eine begrenzte Produktgruppe im Einzelnen ergeben. Die Regulierung umfasst damit sowohl grundlegende Richtungsentscheidungen als auch die Suche nach der besten Lösung im konkreten Anwendungsfall (vgl. zum Gegensatz Jachtenfuchs/Kohler-Koch 2004: 80). Wenn umfassende Regelungen für jeweils eng begrenzte Produktgruppen festgelegt werden, wie dies im Rahmen des EU-Harmonisierungsprogramms bis in die 1980er Jahre hinein geschah, fallen beide Funktionen in einer einzigen Entscheidung zusammen. Wenn die Funktionen jedoch durch unterschiedliche Akteure getrennt voneinander ausgeführt werden, wird ein ursprünglich einheitlicher Entscheidungsprozess in mindestens zwei Teilprozesse zerlegt, die jeweils unterschiedliche Funktionen im Rahmen eines umfassenderen Gesamtprozesses erfüllen. Aus einer differenzierungstheoretischen Sicht beschränkt sich das delegierende Entscheidungssystem auf einen Teil der insgesamt zu bewältigenden Aufgabenlast und weist einen anderen Teil einem neuen Subsystem zu, das dadurch eigene Entscheidungsspielräume gewinnt (Gehring 2005).

Die Bestimmung der allgemeinen Regulierungsziele und die Errichtung der Regulierungsregime fällt in den Aufgabenbereich des politischen Entscheidungsprozesses, der in der Europäischen Union in der Regel auf der Ebene der regulären Rechtsetzung durch Kommission, Rat und Parlament ausgeführt wird. Auf dieser Ebene wird nicht nur darüber entschieden, dass die beiden zentralen Teilfunktionen des Regulierungsprozesses getrennt voneinander erfüllt werden sollen. Da Entscheidungskompetenzen auf andere Akteure übertragen werden, muss in prozeduraler Hinsicht auch festgelegt werden, welche Akteure nach welchen Verfahren auf der neu entstandenen zweiten Ebene entscheiden. Um die inhaltliche Zielbestimmung nicht vollständig auf die Expertenebene zu verlagern, müssen zusätzlich materielle Entscheidungsgrundsätze festgelegt werden. Diese inhaltlichen Vorgaben müssen hinreichend konkret ausfallen, um Entscheidungskriterien für den nachgeordneten Teilprozess darstellen zu können, sollen dort verfügbaren Entscheidungsspielraum aber nicht vollständig beseitigen, weil das nachgeordnete Subsystem dadurch funktionslos werden würde.

Durch die Beschränkung der politischen Ebene auf die Funktionen der Zielbestimmung und der Organisation des Regulierungsregimes wird die Binnenrationalität politischer Entscheidungsprozesse drastisch verändert. Während die Beteiligten einer undifferenzierten Verhandlungsrunde ihr Handeln an ihren jeweils aktuellen, situativen Partikularinteressen ausrichten können, müssen sie nun inhaltliche und verfahrensbezogene Vorgaben ausarbeiten, die den nachgeordneten Entscheidungsprozess und die an ihm beteiligten Akteure insgesamt so lenken, dass die dort getroffenen Anwendungsentscheidungen zu akzeptablen Ergebnissen führen. Dadurch wird die Interessenlage komplexer und diffuser. Zum einen entstehen Konsistenzzwänge, weil ein Mitgliedstaat seine Interessen für ein größeres Regulierungsfeld - etwa für Maschinen, Spielzeug oder Arzneimittel - definieren muss und die Sonderbelange einzelner Produkte nur noch in diesem Rahmen berücksichtigen kann (Brennan/Buchanan 1985: 28-31). Zum anderen müssen angesichts des längerfristigen Bestandes eines institutionalisierten Entscheidungsverfahrens auch zukünftige Probleme in Rechnung gestellt werden, die zum Zeitpunkt der Grundsatzentscheidung noch gar nicht erkennbar sind. Im Idealfall entsteht ein Rawls'scher "Schleier des Nichtwissens“ (Rawls 1998), der die situativen Partikularinteressen verhüllt. Unter diesen Bedingungen werden selbst rationale Egoisten nach Entscheidungsvorgaben streben, die auch unabhängig von situativen Partikular- 
interessen Regulierungsentscheidungen erwarten lassen, die problemadäquat und deshalb grundsätzlich akzeptabel sind.

Die nachfolgenden Anwendungsentscheidungen folgen aufgrund der externen Vorgaben und des Zuschnitts der Verhandlungssituation einer spezifischen Logik, welche die Koordinationslogik einfacher Verhandlungen außer Kraft zu setzen vermag. Erstens konfrontiert der engere Zuschnitt jedes einzelnen Entscheidungsfalls die beteiligten Akteure immer dann mit einer Verhandlungssituation, in der verteilungsorientiertes Verhandeln nicht vorteilhaft ist, wenn ein Interessenausgleich, der alle Beteiligten gegenüber dem Status quo besser stellen würde, aufgrund der Kleinteiligkeit eines Entscheidungsproblems nicht erreichbar ist. Bei isolierter Betrachtung wiirde jede einzelne Entscheidung blockiert werden. Da im Laufe der Zeit eine größere Zahl ähnlicher Entscheidungen zu treffen ist, können die einzelnen Entscheidungssituationen jedoch so zu einer Kette verknüpft werden, dass der klassische Ausweg aus wiederholt auftretenden Gefangenendilemma-Situationen zur Verfügung steht (Axelrod 1984). Es lohnt sich dann, nicht ausschließlich die im Einzelfall bestehenden Partikularinteressen, sondern auch die Folgen des eigenen Verhaltens für zukünftige Kooperationssituationen zu berücksichtigen. Dann wird es sinnvoll, sich gemeinsam auf einen von außen vorgegebenen, allgemein akzeptierten „Orientierungspunkt“ (Schelling 1995: 100) in der Form einer besonders sinnvollen (,vernünftigen“) Umsetzung der inhaltlichen Entscheidungsvorgaben zu bezichen. Zweitens kann der Entscheidungsprozess der nachgeordneten Ebene selbst noch einmal funktional ausdifferenziert werden. So lassen sich die typischen Entscheidungsfunktionen der sachlichen (d. h. wissenschaftlichen oder technischen) Vorbereitung, der eigentlichen Entscheidung und der Kontrolle dieser Entscheidung anhand der verbindlich vorgegebenen Entscheidungskriterien an unterschiedliche Akteure übertragen, sodass ein System der gegenseitigen Beeinflussung und Kontrolle entsteht.

Die Einbindung aller binnenmarktpolitischen Regulierungsentscheidungen in das europäische Rechtssystem stärkt die Verbindlichkeit der inhaltlichen und prozeduralen Vorgaben, die auf der politischen Entscheidungsebene getroffen werden. Wenn Entscheidungsvorgaben der Kontrolle durch den EuGH zugänglich sind, wird dieser automatisch zu einem wichtigen Akteur, dessen latente Präsenz sich auf die Entscheidungsfunktionen der anderen Subsysteme auswirken kann. Inhaltliche Vorgaben, die hinreichend konkret formuliert und in der verbindlichen Form supranationalen Rechts festgelegt sind, schaffen einen impliziten Zwang für alle an der Regulierungsentscheidung beteiligten Entscheidungsgremien, ihre (Teil-)Entscheidungen auf der Grundlage dieser Vorgaben zu rechtfertigen ("giving reasons requirement", Shapiro 1992). Dadurch wird es schwieriger, innerhalb des verbleibenden Entscheidungsspielraums Positionen durchzuseczen, die sich nur schwer begründen lassen. Spezifische Verfahrensvorschriften, die in der Form supranational verbindlichen Rechts vorgegeben sind, tragen dagegen zur Aufrechterhaltung institutioneller Grenzen zwischen den Subsystemen bei und sichern deren Autonomiespielräume (Shapiro 2001).

Funktional differenzierte Entscheidungssysteme steigern die Leistungsfähigkeit von Entscheidungsprozessen, aber sie müssen zuverlässig so integriert werden, dass die einzelnen Teilentscheidungen sich zu einem sinnvollen Ganzen zusammenfügen. Aus arbeitsteiligen Produktionsprozessen ist seit langem bekannt, dass funktionale Differenzierung zu einer erheblichen Leistungssteigerung führen kann, weil jedes beteiligte Sub- 
system sich auf bestimmte Aufgaben spezialisieren und diese deshalb besonders gut auszuführen vermag (Mayntz 1988: 19). Allerdings steigt mit der funktionalen Differenzierung auch die Störanfälligkeit solcher Prozesse. Es bedarf also geeigneter Mechanismen, die verhindern, dass durch die Ausdifferenzierung Entscheidungsblockaden entstehen. Je enger ein ausdifferenzierter Regulierungsprozess an das europäische Rechtssystem angebunden ist, desto stärker werden die für alle Teilsysteme verbindlichen Vorgaben trotz unterschiedlicher Ausrichtung der einzelnen Subsysteme zu einem gemeinsamen Referenzpunkt des Entscheidungsprozesses - und umso stärker wird ein funktional ausdifferenzierter Entscheidungsprozess durch Recht integriert. An die Stelle eines Letztentscheiders, der alle vorangegangenen Teilentscheidungen aufgrund der verfügbaren Machtressourcen aufheben oder modifizieren kann, tritt damit die rechtiche Überprüfung der Teilentscheidungen auf ihre Kompatibilität mit den inhaltlichen und prozeduralen Vorgaben.

Die Entscheidungslogiken funktional differenzierter Regulierungsregime lassen sich nicht durch die Analyse einzelner Teilentscheidungsprozesse erfassen, weil die Wechselwirkungen zwischen den jeweils beteiligten Subsystemen dadurch nicht erkennbar werden. Da keines der beteiligten Subsysteme den gesamten Entscheidungsprozess mehr vollständig kontrolliert, bestimmt auch keines allein die Entscheidungslogik des Gesamtsystems. Diese entsteht vielmehr durch das Zusammenspiel der verschiedenen Entscheidungslogiken der beteiligten Subsysteme. Die in der politik- und rechtswissenschaftlichen Diskussion verbreitete Betrachtung einzelner Komitologie-Ausschüsse oder Regulierungsagenturen greift deshalb zu kurz, weil sie das Zusammenspiel mehrerer Teilprozesse, die zu unterschiedlichen Zeitpunkten an verschiedenen Orten stattfinden, nicht berücksichtigt.

\section{Differenzierte Entscheidungsprozesse im "Neuen Ansatz" der Binnenmarktregulierung}

Der Sachbereich der produktbezogenen Risikoregulierung bildet nicht automatisch eine Domäne der wenig konfliktreichen technischen oder wissenschaftlichen Expertise. Bis in die 80er Jahre hinein wurde die Angleichung von Produktanforderungen im Binnenmarkt auf Ratsebene angestrebt. Die sektorspezifische Vollharmonisierung für kleine Produktgruppen durch detaillierte Richtlinien war aber zähflüssig und auf vielen Gebieten über Jahre hinweg blockiert (Vos 1999: 54-56). Zum einen war die Koordinationsfähigkeit zwischenstaatlicher Verhandlungen trotz der vergleichsweise kleinen Produktbereiche mit der Vielzahl der zu berücksichtigenden technischen Details überfordert. Zum zweiten führten scharfe Interessengegensätze im Rat zu häufigen Blockaden des Entscheidungsprozesses. Die Blockadesituation implizierte, dass die Regulierung zum Schutz von Verbrauchern und Anwendern weiterhin auf nationaler Ebene stattfand.

Im Zuge des Binnenmarktprogramms hat die Europäische Union im Bereich der Regulierung produktbezogener Risiken unter dem Begriff „Neuer Ansarz" systematisch Regulierungsregime eingeführt, die auf dem Grundsatz der funktionalen Differenzierung beruhen. Im Unterschied zu dem alten Ansatz der sektoralen Vollharmonisierung sieht der Neue Ansatz vor, dass nur noch die grundlegenden Sicherheitsanforderungen für große Produktbereiche - etwa für "Maschinen“, "Lebensmittelzusatzstoffe" oder 
„Pharmazeutika“ - in der Form von Richtlinien und Verordnungen des Rates und des Parlaments niedergelegt werden, während Ausführungsbestimmungen auf nachgeordnete Entscheidungsverfahren verlagert werden (Joerges et al. 1988).

Die Zielsetzung des Neuen Ansatzes bestand primär in der Auflösung von Entscheidungsblockaden im Rat. Angestrebr wurde eine Entlastung der politischen Entscheidungsebene von einem erheblichen Teil der insgesamt anfallenden Entscheidungslast, der auf nachgeordnete Entscheidungsprozesse übertragen wurde - nicht die qualitative Verbesserung der Risikoregulierung. Die Verfahren des Neuen Ansatzes bilden heute den Normalfall der produktbezogenen Risikoregulierung im europäischen Binnenmarkt. Nur in wenigen, politisch besonders brisanten Fällen werden europäische Produktanforderungen noch in der Form sektoraler Richtlinien von Rat und Parlament erlassen. Umgekehrt werden ständig neue Produktbereiche - in jüngster Zeit etwa die Chemikalienregulierung - Entscheidungsverfahren unterworfen, die dem Modell des Neuen Ansatzes folgen.

Die konkrete Ausgestaltung der Regulierungsregime ist je nach Produktbereich unterschiedlich. In der Lebensmittelregulierung wurde zunächst auf die Einrichtung von Komitologie-Ausschüssen zurückgegriffen. Die Normungspolitik für technische Produkte nutzt dagegen die Expertise privatrechtlicher Regulierungsagenturen. Daneben treten seit Anfang der 90er Jahre - wie zum Beispiel im Fall der Arzneimittelzulassung - zunehmend selbständige Regulierungsbehörden. Die Ursache für diese Varianz liegt in den unterschiedlichen Ausgangsbedingungen, die zum Zeitpunkt der Einführung des Neuen Ansatzes in den Mitgliedstaaten existierten. Während in einigen Bereichen auf existierende nationale oder europäische Regulierungseinrichtungen zurückgegriffen werden konnte, fehlten diese in anderen Bereichen.

Die instirutionelle Varianz ermöglicht es, die rationalisierende Wirkung unterschiedlicher Ausprägungen des Neuen Ansatzes zu vergleichen. Es ist zu erwarten, dass Entscheidungen umso problemgerechter ausfallen, je stringenter die entstehenden Regulierungsstrukturen zur funktionalen Differenzierung der Entscheidungstätigkeit führen und je stärker alle beteiligten Entscheidungsträger an rechtlich verbindliche Vorgaben gebunden sind. Zwei Dimensionen müssen dafür beachtet werden: Erstens stellt sich die Frage, ob es im einzelnen Produktbereich gelang, eine angemessene Funktionsaufteilung zwischen der politischen und der nachfolgenden, expertisegeprägten Ebene zu finden. Wichtig ist es, dass die politische Ebene bei der Definition von materiellen Zielvorgaben auf Detailausfuhrungen verzichtet, die Ziele aber dennoch hinreichend genau spezifiziert. Nur wenn Zielvorgaben auf der Anwendungsebene operationalisiert werden können, tragen sie zur inhaldichen Ausrichtung der Expertengremien bei. Zweitens ist die Fähigkeit zu problemangemessenen Entscheidungen abhängig von der institutionellen Ausgestaltung auf der Anwendungsebene. Das Verfahren muss so konstruiert sein, dass es die Einhaltung der Zielvorgaben sicherstellt. 


\subsection{Die Zulassung von Arzneimitteln im Agenturverfahren}

\subsubsection{Die Europäisierung der Arzneimittelzulassung}

Während die Herstellung eines einheitlichen Binnenmarktes in den beiden anderen hier untersuchten Politikbereichen durch eine Entscheidungsblockade im Rat behindert wurde, schlug im Bereich der Arzneimittelzulassung das Prinzip der gegenseitigen Anerkennung nationaler Zulassungen fehl. Die nationalen Zulassungsbehörden weigerten sich, Zulassungen anderer Mitgliedstaaten anzuerkennen, da sie sonst die Verantwortung für von ihnen selbst nicht geprüfte Produkte hätten übernehmen müssen (Glaeske et al. 1988; Vos 1999: 210). Stattdessen überprüften die nationalen Behörden jeden Zulassungsantrag erneut und kamen dabei oftmals zu unterschiedlichen Ergebnissen.

Um die Blockade der gegenseitigen Anerkennung im Arzneimittelsektor aufzuheben, wurde zu Beginn der 1990er Jahre die Zulassung von hoch innovativen Arzneimitteln in der EU zentralisiert. ${ }^{1}$ Für diese Produkte wurde ein Zulassungsverfahren errichter, an dessen Ende eine einheitliche EU-Zulassungsentscheidung steht. Innerhalb dieses zentralisierten Zulassungsverfahrens wird die wissenschaftliche Bewertung von Arzneimitteln an eine neu eingerichtete europäische Arzneimittelagentur delegiert. Es findet also eine vertikale Differenzierung zwischen Normsetzung - auf der Ebene regulärer Gesetzgebung durch Rat und Parlament - und Normanwendung - auf der Ebene des zentralisierten Verfahrens unter Leitung der europäischen Arzneimittelagentur statt.

Die besondere Form dieses supranationalen Regulierungsregimes für Arzneimittel ist auf die starke Stellung nationaler Zulassungsbehörden zurückzuführen (Krapohl 2007). Seit der Contergan-Katastrophe waren nahezu alle Mitgliedstaaten der EU dazu übergegangen, Zulassungsentscheidungen für Arzneimittel durch mehr oder weniger unabhängige Behörden vornehmen zu lassen. Diese Behörden konnten sich erfolgreich gegen die gegenseitige Anerkennung zur Wehr setzen. Um ihren Widerstand zu überwinden, wurden sie schließlich in das neue EU-Regime mit einbezogen. Den Kern der europäischen Arzneimittelagentur bildet damit ein Netzwerk nationaler Behörden (Majone 1997). Über die Zulassungsanträge wird zwar gemeinsam entschieden, die Bewertung der Anträge erfolgt jedoch nach wie vor dezentral durch nationale Behörden.

\subsubsection{Die starke Verrechtlichung der europäischen Arzneimittelzulassung}

Wegen der besonderen Entwicklung der europäischen Arzneimittelzulassung ist der Politikbereich hochgradig verregelt und verrechtlicht. Seit dem Contergan-Skandal in den 1960 er Jahren hatte die Kommission versucht, das Prinzip der gegenseitigen Anerkennung von Arzneimitteln durch eine Harmonisierung der Zulassungsvoraussetzungen zu

1 Im Gegensatz zu der zentralisterten Zulassung von hoch innovativen Arzneimitteln werden weniger innovative Produkte immer noch in einem Verfahren der gegenseitigen Anerkennung zugelassen. Jedoch findet im Fall der Nicht-Anerkennung der ursprünglichen Zulassungsentscheidungen durch die betroffenen Mitgliedstaaten ein bindendes Schiedsverfahren statt, das im Wesentlichen dem zentralisierten Zulassungsverfahren gleicht. 
unterstïtzen (Feick 2000). Mit der Vereinheitlichung der substanziellen Vorgaben für Arzneimittel und Zulassungsanträge sollte der Handlungsspielraum der nationalen Behörden soweit eingeschränkt werden, dass eine gegenseitige Anerkennung von Zulassungen unproblematisch geworden wäre. Die detaillierten Zulassungskriterien wurden ohne Änderungen in das neue Regulierungsregime übernommen und sind stetig weiterentwickelt worden - zuletzt in einem Reformprozess zu Beginn des neuen Jahrtausends.

Die substanziellen Vorgaben für die Zulassung von Arzneimitteln untersagen den beteiligten Akteuren im zentralisierten Zulassungsverfahren, Entscheidungen auf der Grundlage von wirtschaftlichen oder politischen Erwägungen zu fällen. Arzneimittel dürfen nur auf der Basis der drei Kriterien der Wirksamkeit, Unbedenklichkeit und Qualität zugelassen werden. Um die Einhaltung dieser drei Kriterien zu überprüfen, muss jedes Arzneimittel eine Reihe vorklinischer und klinischer Tests absolvieren, deren Ergebnisse in einem Zulassungsantrag protokolliert werden. Mit diesen strengen Vorgaben steuern die Akteure der politischen Ebene - also die Kommission, die Mitgliedstaaten und seit Kurzem auch das Europäische Parlament - den Zulassungsprozess, ohne in Einzelfallentscheidungen einzugreifen. Jedoch binden die politischen Akteure nicht nur die europäische Arzneimittelagentur, sondern auch sich selber. Da bis auf das Parlament alle gesetzgebenden Akteure in das Zulassungsverfahren involviert sind, sind sie von ihren eigenen substanziellen Entscheidungsvorgaben gleichermaßen betroffen.

\subsubsection{Checks-and-Balances bei der europäischen Zulassung von Arzneimitteln}

Die Europäische Arzneimittelagentur ist in ein funktional differenziertes Entscheidungsverfahren eingebunden. Innerhalb des zentralisierten Zulassungsverfahrens erarbeiter der Expertenausschuss der Agentur, der aus Vertretern der nationalen Zulassungsbehörden besteht, auf Antrag der Hersteller wissenschaftliche Gutachten über die Zulassungsfähigkeit von Arzneimitteln, aber er trifft die eigentlichen Entscheidungen nicht selbst. Auf der Grundlage der Gutachten erstellt die Kommission einen Entscheidungsentwurf, der dann einem Komitologie-Ausschuss vorgelegt wird. Formell muss eine qualifizierte Mehrheit der Mitgliedstaaten einem Entscheidungsvorschlag der Kommission zustimmen, der andernfalls dem Rat zur letztendlichen Entscheidung vorgelegt wird.

Trotz der formal strikten politischen Kontrolle durch die Kommission und die Mitgliedstaaten entwickelte sich die europäische Arzneimittelagentur zu einem extrem starken Agenda-Setter im zentralisierten Zulassungsverfahren (Krapohl 2005). Nach einer 2001 durchgeführten Bestandsaufnahme der Kommission wurde bis dahin jedes Gutachten der Agentur von der Kommission als Entscheidungsvorschlag übernommen, nur ein Bruchteil von 3,5 Prozent dieser Vorschläge wurde Gegenstand einer Auseinandersetzung im Mitgliedstaatenausschuss und keine einzige Entscheidung wurde an den Rat weitergegeben. Somit entsprachen die letztendlichen Zulassungsentscheidungen ohne Ausnahme den Gutachten der Arzneimittelagentur.

Der ungewöhnlich starke Einfluss der Agentur kann vor allem auf die starke Verrechtlichung des Regulierungsbereichs zurückgeführt werden. Die substanziellen Zulassungskriterien stärken innerhalb des Zulassungsverfahrens vor allem die Agentur, da 
Entscheidungen aus wirtschaftlichen und politischen Erwägungen heraus nicht zugelassen sind. Verstärkt wird dieser Effekt durch die mögliche Überprüfung von Entscheidungen durch den europäischen Gerichtshof und das Gericht erster Instanz. Da die Antragsteller auf Arzneimittelzulassungen von den Entscheidungen der Kommission unmittelbar und individuell betroffen sind, genießen sie einen besonderen Rechtsschutz und sind grundsätzlich berechtigt, vor den europäischen Gerichten Klage zu erheben (Collatz 1996). Damit werden die europäischen Gerichte zu potenziellen zusätzlichen Akteuren im zentralisierten Zulassungsverfahren.

Trotz ihrer starken Stellung im zentralisierten Zulassungsverfahren kann die europäische Arzneimittelagentur keinesfalls unabhängig und willkürlich über Zulassungen entscheiden (Gehring/Krapohl 2007). Im Gegenteil, sie wird durch mindestens drei Mechanismen kontrolliert: Erstens können natürlich die Kommission und die Mitgliedstaaten nach wie vor einschreiten, falls die Agentur von den substanziellen Entscheidungsvorgaben abweicht. Zweitens können aber auch private Akteure - vor allem die Antragsteller - die Agentur auf gerichtlichem Wege für ihre Entscheidungen zur Verantwortung ziehen. Und drittens kontrollieren sich innerhalb der Agentur auch noch die Experten der nationalen Zulassungsbehörden gegenseitig bei der Evaluation der Zulassungsanträge. Entscheidend ist, dass keiner der drei Kontrollmechanismen die Arbeit des ganzen Regimes dominieren kann, sondern dass sie sich gegenseitig ausbalancieren. Das Regime bleibt somit unter Kontrolle, obwohl es von keiner Seite alleine kontrolliert werden kann (Majone 1997).

\subsubsection{Die Bewertung des zentralisierten Zulassungsverfahrens}

Das zentralisierte Zulassungsverfahren hat einen wichtigen Beitrag zur Errichtung eines einheitlichen Binnenmarktes für Arzneimittel geleistet (Vogel 1998; Feick 2002). Zulassungen, die aus diesem Verfahren resultieren, sind in allen Mitgliedstaaten gültig. Damit wird der europäische Arzneimittelmarkt nicht mehr durch unterschiedliche Entscheidungspraktiken nationaler Zulassungsbehörden behindert. Das zentralisierte Verfahren wurde in einem Evaluationsbericht von 2000 von der europäischen Pharmaindustrie sehr positiv bewertet (CMS 2000).

Auch hinsichtlich des Verbraucher- und Gesundheitsschutzes scheint das EU-Regulierungsregime für Arzneimittel seine Ziele zu erreichen. Der häufig geäußerte Verdacht, dass die europäische Arzneimittelregulierung einseitig die Interessen der pharmazeutischen Industrie bevorzuge (Abraham/Lewis 2001: Permanand/Mossialos 2005), bestätigt sich nicht. In dem genannten Evaluationsbericht wird das zentralisierte Zulassungsverfahren von Patienten- und Berufsverbänden positiver bewertet als das Verfahren der gegenseitigen Anerkennung, in dem die Mitgliedstaaten für den Schutz ihrer Verbraucher zuständig sind. Ein wichtiger Grund für den Erfolg des zentralisierten Verfahrens dürften die verschiedenen rechrlichen und politischen Kontrollmechanismen sein, welche die Agentur bei ihrer Tätigkeit überwachen.

Aufgrund dieser positiven Beurteilungen durch Industrie und Verbraucher sind in einem Reformprozess zu Beginn des neuen Jahrtausends zentrale Elemente des Regimes verstärkt worden (Broscheid/Feick 2005). Der Anwendungsbereich des zentralisierten Verfahrens wurde auf weitere Arzneimittel ausgeweitet und die Unabhängigkeit der 
Agentur wurde weiter gestärkt. Insgesamt kann dieser Reformprozess als Bestätigung eines erfolgreichen Verfahrens supranationaler Risikoregulierung gewertet werden.

\subsection{Die Normung technischer Güter im europäischen Binnenmarkt}

\subsubsection{Die Europäisierung der Normung technischer Güter}

Vor Einführung des Neuen Ansatzes im Rahmen des Binnenmarktprogramms war die Harmonisierung technischer Produktanforderungen in der Europäischen Union in weiten Teilen blockiert. Ab 1969 versuchte die EU, den freien Warenverkehr technischer Produkte durch eine vollständige Harmonisierung von Produktvorschriften zu gewährleisten. Im Rahmen des üblichen EU-Gesetzgebungsverfahrens wurden detaillierte Richtlinien ausgearbeitet, welche einheitliche Anforderungen für sehr kleine Produktgruppen enthielten, z. B. für die Abschleppeinrichtungen bei landwirtschaftlichen Zugmaschinen. Für die Blockade des Verfahrens gab es zwei Gründe: Zum einen überlasteten die technischen Details die Gesetzgebung (Pelkmans 1987: 251). Zum anderen versuchten viele Mitgliedstaaten, ihre nationalen Produktanforderungen auf die europäische Ebene zu übertragen.

Ein Instrument zur Auflösung der Blockade fand die EU in der Methode der „Verweisung auf technische Normen “, die auch nationale Normungsregime kennzeichnet. Finige Nationalstaaten der EU begegneten dem Problem der mangelnden Expertise des Gesetzgebers in technischen Dingen, indem sie auf Gesetzesebene nur sehr allgemeine Sicherheitsanforderungen oder prozedurale Regeln definieren, für die Definition produktspezifischer Regelungen aber auf die technischen Normen der nationalen Normungsbehörden verwiesen.

Indem die Europäische Union die Methode der „Verweisung auf technische Normen" übernahm, führte sie ein funktional differenziertes Verfahren nach der Logik des Neuen Ansatzes ein: Der europäische Gesetzgeber behält sich vor, im Rahmen rechtsverbindlicher Richtlinien die allgemeinen Gesundheits- und Sicherheitsanforderungen zu definieren. Die Aufgabe der technischen Spezifizierung delegiert er an die privatrechtlichen europäischen Normungsorganisationen CEN (Comité Européen de Normalisation, gegründet 1961), CENELEC (Comité Européen de la Normalisation Electrotechnique, 1974) und ETSI (European Telecommunication Standards Institute, 1988) (Joerges et al. 1988, 360-364). Der Grund für die Einbindung der privatrechtlichen Normungsinstitute in das Verfahren liegt im Ausgangszustand der 80er Jahre begründet: Der Kommission fehlten Fachkenntnisse in der Normung und die europäischen Normungsorganisationen CEN und CENELEC waren schon vorhanden. Sie erschienen als legitime Akteure, da sie sich aus den nationalen Normungsorganisationen zusammensetzen, die in den Mitgliedstaaten (wie zum Beispiel DIN in Deutschland, ÖNORM in Österreich) die Normungsarbeit vornehmen. 


\subsubsection{Verrechtlichung der Regulierung technischer Produkte}

Die Einführung des Neuen Ansatzes ermöglichte es, im Rahmen einer EU-Richtlinie nicht mehr nur einzelne Produkte, sondern sehr große Produkrbereiche zu regulieren beispielsweise Maschinen oder Spielzeug. Wegen der Beschränkung auf grundlegende Sicherheits- und Gesundheitsanforderungen reduzierte sich die Menge an Regeln, die zur Regulierung eines Produktfeldes nötig sind, drastisch. Selbst im Vergleich zum Fall der Arzneimittelzulassung (Abschnitt 3.1) ist die Verrechtlichung des Bereichs der technischen Normung aber immer noch relativ hoch. Die EU-Richtlinien der politischen Ebene gehen zwar nicht in letzte technische Details, decken aber einzelne Gefahren ab, die von Produkten ausgehen können. In den Anhängen der Maschinenrichtlinie (89/ 392/EWG) wird beispielsweise auf Gefahren durch scharfe Ecken, Kanten, Gase und Flüssigkeiten eingegangen (Berghaus/Langner 1998). Die technischen Vorgaben sind vergleichsweise genau spezifiziert, weil die Richtlinien auch direkt, also ohne zusätzliche Normen, auf die einzelnen Produkte anwendbar sein müssen (Voelzkow 1996, 277-281): Da die EU-Staaten den freien Warenverkehr für alle Produkre gewähren müssen, die die verbindlichen Vorgaben der Richtlinien erfüllen, muss für jede Säge und jede Spielzeugpistole festgestellt werden können, ob sie der Richtlinie genügt.

Der Verzicht auf eine noch weiter gehende Regulierung technischer Details hatte zwei Effekte: Erstens wurde die Blockade der Harmonisierung aufgelöst. Ingesamt gibt es heute 24 technische Richtlinien, die im Vergleich zu früher in relativ kurzer Zeit beschlossen wurden (Vos 1999: 308). Zweitens ist das Sicherheitsniveau der meisten Richtlinien relativ hoch (Berghaus/Langner 1998: 1). Auflösung der Blockade und hohes Schutzniveau lassen sich dadurch erklären, dass sich die Geserzgeber in vielen Fällen aufgrund der Größe des Produktfeldes und der unbegrenzten Gültigkeitsdauer der Regulierung in einer Situation befanden, in der es nicht möglich war, nationale Einzelinteressen einzubringen. Formulierungen, dass Produkte "sicher" sein sollen oder - wie im Fall der Maschinenrichtlinie - von Ecken und Kanten keine Gefahren ausgehen dürfen, machen es schwer, nationale Positionen zu vertreten.

\subsubsection{Zurückdrängung partikularer Interessen im Normungsverfahren}

Die europäische Normung erfolgt im Rahmen eines mehrstufigen, funktional differenzierten Verfahrens, in dem ein Teil der Entscheidungslast von privatrechtlichen Normungsagenturen wahrgenommen wird und staarlichem Einfluss sehr weit entzogen ist. Die Mitgliedstaaten und die Kommission haben sich aber bedeutende Entscheidungsfunktionen zurückbehalten: Das Normungsverfahren beginnt mit einem Auftrag (Mandat), in dem die Kommission nach der Konsultation von Mitgliedstaaten-Ausschüssen die Normungsorganisation mit der Ausarbeitung technischer Normen betraut. Die inhaltliche Erarbeitung der Norm nehmen dann allein die privaten Normungsorganisationen vor. Anschließend wird die Norm von der Kommission geprüft und gegebenenfalls als "harmonisierte Norm“ im Amtsblatt veröffentlicht. Durch diesen Schritt werden die Mitgliedstaaten faktisch verpflichtet, Produkte, die der betreffenden Norm entsprechen, auf ihren Märkten zuzulassen (Konformitätsvermutung). Bezweifelt ein Mitgliedstaat die Vereinbarkeit einer bereits akzeptierten Norm mit der übergeordneten 
Richtlinie, kann er im Rahmen eines Komitologie-Ausschusses ein "Schutzklauselverfahren" anstrengen, das zur Revision der Norm oder zur Rücknahme der Konformitätsvermutung führen kann.

Die Experten der privatrechtichen Normungsorganisationen sind in der Erarbeitung technischer Normen zwar frei von politischer Intervention; institutionelle Mechanismen stellen aber sicher, dass sie nicht völlig beliebig entscheiden können. Erstens wird bereits innerhalb der Normungsorganisationen ein mehrstufiges Verfahren durchlaufen, welches sicherstellt, dass die Argumente verschiedener Interessengruppen gehört werden (Vos 1999: 298-301). Zweitens muss die Normungsorganisation die Mandate der Kommission beachten, die eindeutig auf die Kriterien der Richtlinien verweisen (Stefanova 2005: 271). Drittens bindet die Kontrolle der Norm durch die Kommission die Normungsorganisationen an die Richtlinien und Mandate, da sie bei Verstößen damit rechnen müssen, dass ihre Normentwürfe nicht akzeptiert werden (Voelzkow 1996: 292). Schließlich erfüllt das Schutzklauselverfahren eine dauerhafte Kontrollfunktion. Erfüllt eine Norm die Vorgaben der betreffenden Richtlinie nur unzureichend und erleiden Verbraucher oder Anwender der regulierten Produkte deshalb Schäden, so wird die Norm auf Betreiben eines Mitgliedstaates Gegenstand einer Überprüfung (Vos 1999: 307).

Das Design des Schutzklauselverfahrens beugt der Gefahr vor, dass es von einzelnen Staaten missbraucht wird, um aufgrund partikularer Interessen - etwa zum Zweck des Protektionismus - gültige Normen zu unterlaufen. Ein Schutzklauselverfahren kann von den Staaten über den Komitologie-Ausschuss „Normen und technische Vorschriften" oder über richtlinienspezifische Ausschüsse initiiert werden. Zwei Mechanismen verhindern eine ungehinderte Interessenverfolgung. Erstens muss der an der Prüfung der Norm interessierte Staat die anderen Staatenvertreter von der Notwendigkeit einer Revision überzeugen. In der Praxis legen die Mitglieder dazu umfangreiche technische Informationen und Unfalldaten vor (Stefanova 2005: 264). Zweitens handel es sich bei den Gremien um Beratungsausschüsse, die nicht selbst über die Revision der Norm entscheiden können. Die Entscheidung trifft die Kommission. Ein interessierter Mitgliedstaat muss also nicht nur die anderen Mitgliedstaaten, sondern auch die Kommission von seinem Anliegen überzeugen. Entscheidungsvorschläge, die der Richtlinie widersprächen, würde die Kommission nicht akzeptieren, weil sie dann mit einer Klage vor dem EuGH rechnen müsste.

Die Anwendung des Neuen Ansatzes in der technischen Normung wird heute als großer Erfolg angesehen (Vos 1999: 308). Die Produktrichtlinien werden durch eine große Anzahl an Normen spezifiziert - bei der Maschinenrichtlinie sind es derzeit über 400 (Abl. C 180 v. 2.8.2006: 11-64). Dass das Normungsverfahren die beteiligten Akteure wirksam an die Vorgaben der Richtinien bindet, zeigt die geringe Zahl der Schutzklauselverfahren. Analysen von Einzelfällen zeigen, dass eine Normrevision nur bei Sicherheitsmängeln, aber nicht aus nationalen Partikularinteressen zustande kommt (Stefanova 2005: 271, 275-310) 


\subsubsection{Probleme der Normung: lückenhafre Richtlinien}

Probleme treten bei der technischen Normung dann auf, wenn bei der Ausarbeitung der Richtlinien Lücken entstanden sind. Lücken in der Richtlinie können auf verschiedene Weise zustande kommen, zum Beispiel wenn sich die Akteure im Gesetzgebungsverfahren auf Grund divergierender Interessen nicht auf Kriterien einigen können. Damit übertragen sie den Konflikt auf die nachgelagerte, durch Expertise geprägte Ebene. Dies hat zur Folge, dass den Expertengremien Kriterien für sachgerechte Entscheidungen fehlen, so dass sich große Entscheidungsspielräume auftun. Bei weit auseinanderliegenden Positionen kann es dann vorkommen, dass eine Einigung nur durch taktisches Verhalten und Kompromissbildung zwischen den Experten möglich wird.

Die Probleme, die durch fehlende Vorgaben hervorgerufen werden, illustriert der Fall der Regulierung von Kinderspielzeug. Die europäische Spielzeugrichtlinie (88/378/ EWG) ist in einigen Bereichen unvollständig. Sie enthält zum Beispiel keine Aussagen über Lärm oder organisch-chemische Verbindungen. Der Grund für dieses Defizit sind nationale Einzelinteressen, die während des Gesetzgebungsverfahrens eine Einigung in diesen Bereichen verhindert haben. Die Lücke in der Richtlinie erwies sich für die technische Normung als sehr problematisch: Sie öffnete große Entscheidungsspielräume und ermöglichte interessegeleitetes Verhandeln auch auf der eigentlich expertisegeprägten Normungsebene (Kerler 2005).

\subsection{Die Lebensmittelregulierung im Komitologieverfahren}

\subsubsection{Die Europäisierung der Lebensmittelregulierung}

Ähnlich wie in dem Gebiet der technischen Normung wurde auch im Bereich der Lebensmittelregulierung die Errichtung eines einheitlichen Binnenmarktes lange Zeit durch Entscheidungsblockaden im Rat verhindert. Bis zur Einführung des Neuen Ansatzes versuchte die EU, einen einheitlichen Binnenmarkt für Lebensmittel durch die vollständige Harmonisierung von Produktstandards zu erreichen (Nentwich 1995). Die Mitgliedstaaten befanden sich innerhalb des Rates in einem klassischen Verhandlungsdilemma, weil sie in einem undifferenzierten Verhandlungssystem zeitgleich über komplexe Sachfragen und die Verteilungsfolgen für ihre jeweiligen Industrien verhandeln mussten. Dies führte oft zu unbefriedigenden Kompromissen und erheblichen Verzögerungen der Gesetzgebung.

Zur Auflösung dieser Blockade im Rar entwickelte die Europäische Kommission im Zusammenhang mir dem Binnenmarkrprogramm und parallel zum Bereich der technischen Normung einen neuen Ansatz zur Beseitigung nicht-tarifärer Handelshemmnisse, in dem sie selbst sowie Komitologie-Ausschüsse die zentrale Rolle spielen. Da in den meisten Mitgliedstaaten (Ausnahme sind die skandinavischen Länder) keine unabhängigen Behörden für Lebensmittelregulierung existierten, konnten diese auch nicht in ein europäisches Regulierungsnetzwerk eingebunden werden. Lebensmittelregulierung fand schon auf nationaler Ebene in den jeweiligen Ministerien statt und somit dominierten die Ministerialbürokraten auch die europäische Lebensmittelregulierung in den Komitologie-Ausschüssen (Krapohl 2007). 
Wie die Regulierungsregime der Arzneimittelzulassung und der Normung technischer Güter war auch das Regulierungsregime für Lebensmittel vertikal und horizontal differenziert. In Bezug auf die vertikale Differenzierung war vorgesehen, dass nur noch allgemeine Sicherheitsanforderungen für Lebensmittel im regulären Rechtsetzungsverfahren vereinheitlicht werden sollten, während Detailentscheidungen auf die Anwendungsebene übertragen wurden. Diese Ebene war überdies horizontal differenziert. Zum einen errichtete die Kommission eine Reihe wissenschaftlicher Ausschüsse, die sie zu wissenschaftlichen Aspekten von Regulierungsentscheidungen beraten sollten. Zum anderen wurde die Kommission im Auftrag der Mitgliedstaaten durch KomitologieAusschüsse kontrolliert. Demnach musste die Kommission jeden Entscheidungsvorschlag einem relevanten Mitgliedstaatenausschuss vorlegen, der den Vorschlag entweder annehmen oder an den Rat weitergeben konnte.

\subsubsection{Der Patchwork-Charakter des europäischen Lebensmittelrechts}

Durch die wiederholten Versuche einer vollständigen Harmonisierung von einzelnen Lebensmittelstandards bildete sich ein "Patchwork" (Héritier 1996) europäischer Lebensmittelgesetzgebung heraus. Die EU-Gesetzgebung zur Sicherheit und Qualität von Lebensmitteln reagierte nur ad hoc auf Probleme des Verbraucher- und Gesundheitsschutzes, sofern diese mit dem Ziel eines Binnenmarktes im Zusammenhang standen. Daher fehlten dem europäischen Lebensmittelrecht einheitliche Rechtsnormen zum Verbraucher- und Gesundheitsschutz. Einige Produkte, wie zum Beispiel Schokolade und Schokoladenerzeugnisse, waren hochgradig reguliert, wohingegen in anderen Bereichen, wie zum Beispiel dem Veterinärwesen, nur rudimentäre Vorgaben für den innergemeinschaftlichen Handel existierten.

Die fehlenden inhaltlichen Vorgaben in weiten Teilen der europäischen Lebensmittelregulierung hatten zur Folge, dass die Gesetzgebung weitreichende Ermessensspielräume für Anwendungsentscheidungen offen ließ. Dadurch wurde der Entscheidungsprozess nur unvollständig vertikal differenziert, da die untere Ebene fast die komplette Entscheidungslast alleine zu tragen hatte. Auch eine effektive horizontale Differenzierung auf der unteren Ebene blieb weitestgehend aus, da sich die Komitologie-Ausschüsse aufgrund ihrer formal starken Entscheidungsbefugnisse gegenüber den Akteuren mit wissenschaftlichem Sachverstand durchsetzen konnten. Die MitgliedstaatenAusschüsse und der Ministerrat blieben in ihren Entscheidungen weitgehend frei und konnten von den Fmpfehlungen der wissenschaftlichen Ausschüsse und der Kommission abweichen, da sie ihre Beschlüsse nicht vor dem Hintergrund starker Normen rechtfertigen mussten.

\subsubsection{Die Dominanz der Mitgliedstaaten am Beispiel der BSE-Regulierung}

Die fehlenden inhaltlichen Vorgaben in der europäischen Lebensmittelregulierung stärkten vor allem die Komitologie-Ausschüsse und den Rat, die in der Lage waren, mit ihren Interessen das Entscheidungsverfahren zu dominieren. Im Gegenzug blieben die wissenschaftlichen Ausschüsse und die gerichtliche Überwachung des Regulierungs- 
prozesses schwach. Die Mitgliedstaaten konnten die Empfehlungen der wissenschaftlichen Gremien und der Kommission übergehen, weil sie in ihren Entscheidungen nicht durch inhaltliche Vorgaben gebunden waren. Und eine gerichtliche Kontrolle dieser Entscheidungen war kaum möglich, da der Ermessensspielraum auf der Anwendungsebene sehr groß war. Es entstand also kein System gegenseitiger Kontrolle, sondern eine einseitige Dominanz der Mitgliedstaaten.

Die Dominanz der Mitgliedstaaten wurde während der Regulierung der Rinderseuche BSE besonders deutlich (Krapohl/Zurek 2006). In der ersten Zeit nach der Entdeckung von BSE bei britischen Rindern im Jahr 1986 herrschte erhebliche Unsicherheit über die Gefahren, die von dem Verzehr BSE-verseuchten Rindfleisches für die europäischen Verbraucher ausgingen. Diese wissenschaftliche Unsicherheit wurde von britischen Landwirtschaftsinteressen genutzt, um die Hypothese einer Nicht-Übertragbarkeit von BSE auf Verbraucher aktiv zu vertreten (Dressel 2002; Millstone/van Zwanenberg 2001). Als die britische Regierung dann im März 1996 zugeben musste, dass der Verzehr von BSE-verseuchtem Rindfleisch in der Tat zu einer Infektion des Verbrauchers mit einer neuen Variante der Creutzfeld-Jakob-Krankheit führen könnte, erließen die anderen, vordergründig nicht betroffenen Mitgliedstaaten ein Exportverbot für britisches Rindfleisch auf den europäischen Binnenmarkt (Westlake 1997). Gleichzeitig waren die anderen Mitgliedstaaten jedoch nicht bereit, neben dem Exportverbot für britisches Rindfleisch weitere substanzielle Maßnahmen zur Bekämpfung der Seuche zu erlassen. Maßnahmen die vom wissenschaftlichen Lenkungsausschuss vorgeschlagen wurden, wurden vom ständigen Veterinärausschuss und dem Rat wiederholt zurückgewiesen. Eine gemeinsame, EU-weite Bekämpfung der Seuche wurde erst möglich, als im Jahr 2000 deutlich wurde, dass sich die Rinderseuche bereits auf nahezu alle Mitgliedstaaten ausgebreitet hatte.

Die Dominanz der Mitgliedstaaten auf der Ebene der Anwendungsentscheidungen und die fehlenden inhaltichen Vorgaben führten dazu, dass auch hier Entscheidungsblockaden auftraten. Bis 1996 war Großbritannien in der Lage, sich gegen ein Exportverbot zur Wehr zu setzen, und nach 1996 blockierten die anderen Mitgliedstaaten gemeinsame Maßnahmen gegen die Rinderseuche. Wie vor der Einführung des Neuen Ansatzes im Rat waren die Mitgliedstaaten nun auch innerhalb der Komitologie-Ausschüsse mit Einzelfallentscheidungen konfrontiert und in ihrem Entscheidungsspielraum nicht durch inhaltliche Vorgaben eingeschränkt. Die funktionale Differenzierung des Neuen Ansatzes verfehlte ihre Wirkung, da nahezu die ganze Entscheidungslast an die untere Ebene delegiert wurde.

\subsubsection{Die Bewertung des Komitologieverfahrens im Lebensmittelscktor}

Im Fall der BSE-Regulierung war das EU-Ausschusswesen im Lebensmittelsektor weder in der Lage, die europäischen Verbraucher adäquat vor der Rinderseuche zu schützen, noch eine Ausbreitung derselbigen über die Landesgrenzen Großbritanniens hinaus zu verhindern. Dieses Versagen im BSE-Fall führte in den 90er Jahren zu einer erheblichen Vertrauenskrise der Verbraucher in die Sicherheit ihrer Lebensmittel und die Regulierungskapazitäten der EU (Ansell/Vogel 2006; Majone 2000). Am Ende musste das EU-Regulierungsregime für Lebensmittel grundsätzlich reformiert werden. Im Jahr 
2002 wurde eine europäische Behörde für Lebensmittelsicherheit geschaffen, die die Kommission nun anstelle der wissenschaftlichen Ausschüsse berät. Überdies wurde ein rudimentäres Lebensmittelgesetz verabschiedet, auf dessen Grundlage die Kommission nun handeln kann. Jedoch ist es nach wie vor fraglich, ob diese Behörde und dieses Lebensmittelgesetz ausreichen, um die Probleme der europäischen Lebensmittelregulierung in Zukunft zu lösen (Krapohl/Zurek 2006).

Das Versagen des Ausschusswesens ist aufgetreten, obwohl das Entscheidungsverfahren zur Lebensmittelregulierung formal sowohl vertikal als auch horizontal differenziert war. Zum einen wurden Kompetenzen von der Ebene der regulären Gesetzgebung auf eine nachgeordnete Ebene übertragen, und zum anderen wurden die Kommission, die Mitgliedstaaten-Ausschüsse und die wissenschaftlichen Ausschüsse in einen differenzierten Entscheidungsprozess eingebunden. Da jedoch keine kohärenten inhaltlichen Vorgaben existierten, entstand kein System der Checks-and-Balances auf der Anwendungsebene. Somit waren alle Akteure in ihren Entscheidungen relativ frei. Die eigentliche Entscheidungsmacht sammelte sich daher im Komitologie-Ausschuss und im Ministerrat, wo die Mitgliedstaaten dann doch wieder in einem relativ undifferenzierten Verhandlungssystem über ihre Partikularinteressen verhandelten.

Eine stärkere Verteilung der Entscheidungstätigkeit auf beide Ebenen und damit auch eine stärkere Verrechtlichung der europäischen Lebensmittelregulierung hätten das Ausschusswesen wirksam gegen ein solches Versagen absichern können. Wenn die obere Ebene kohärente und systematische Vorgaben für einzelne Regulierungsentscheidungen festgelegt hätte, wäre die untere Ebene daran gebunden gewesen und hätte vom EuGH überwacht werden können. Die Mitgliedstaaten hätten in diesem Fall den Entscheidungsprozess nicht mit ihren kurzfristigen Partikularinteressen dominieren können und Entscheidungsblockaden wären somit wesentlich unwahrscheinlicher geworden.

\section{Fazit}

Auf dem Gebiet der europäischen Binnenmarktpolitik ist in den vergangenen zwanzig Jahren das Modell eines Regulierungsregimes entstanden, das nicht nur die Entscheidungsfähigkeit des supranationalen Regulierungsapparates sicherstellt, ohne staatliche und nicht-staatliche Träger partikularer Interessen vom Entscheidungsprozess auszuschließen, sondern auch problemadäquate Regulierungsergebnisse verspricht. Die Blockade des politischen Entscheidungsprozesses wird durch die Entlastung von Detailaufgaben und die Beschränkung auf übergreifende Steuerungsfunktionen aufgelöst. Dadurch kann technisch-wissenschaftliche Expertise eine zentrale Rolle übernehmen, ohne selbst die politische Zielsetzung festlegen zu müssen.

Die konkrete Ausgestaltung des Regulierungsregimes in den drei untersuchten Fällen der Regulierung von Produktrisiken im europäischen Binnenmarkt unterscheidet sich deutlich voneinander und lässt sich wesentlich auf die unterschiedlichen mitgliedstaatlichen Ausgangsbedingungen in den Regulierungsbereichen zurückführen. Wo starke Regulierungsbehörden auf der nationalen Ebene bestehen, wie im Bereich der Arzneimittelzulassung, werden sie innerhalb administrativer Regulierungsnetzwerke auch maßgeblich an der europäischen Regulierung beteiligt. Wo die Mitgliedstaaten bereits auf private beziehungsweise halbstaatliche Standardsetzung zurückgreifen, wie im Be- 
reich der Produktnormung, werden die entsprechenden Einrichtungen im Sinne eines nicht-staatlich organisierten Regulierungsnetzwerkes an der Regulierung beteiligt. Und wo sowohl staatliche Fachbehörden als auch nicht-staatliche Regulierungseinrichtungen in den Mitgliedstaaten fehlen, wie im Bereich der Lebensmittelkontrolle, übernehmen die in den Komitologie-Ausschüssen vertretenen politisch-administrativen Repräsentanten der zuständigen Fachministerien eine zentrale Rolle im Regulierungsprozess. Die im Rahmen der Mitgliedstaaten jeweils vorherrschende Organisationsform wird damit weitgehend auf die europäische Ebene übertragen.

Die dem Neuen Ansatz folgenden Regime zur Produktregulierung haben drei Folgen für das Regieren in der Europäischen Union. Erstens tragen sie zur Schaffung eines Binnenmarktes in den betroffenen Bereichen bei. Zweitens verlagern sie die Regulierung zum Schutz von Verbrauchern und Anwendern der betreffenden Produkte von der mitgliedstaatlichen auf die europäische Ebene. Damit entstehen europäische Produktsicherheits- beziehungsweise Verbraucherschurzpolitiken mit marktkorrigierender Wirkung, die den Binnenmarkt flankieren. Drittens verlagern die eingerichteten Verfahren einen unterschiedlich großen Anteil der Regulierungstätigkeit von der politisch legitimierten europäischen Gesetzgebungsebene, an der Kommission, Rat und Parlament beteiligt sind, auf eine durch Expertise geprägte nachgeordnete Entscheidungsebene.

Die Regulierungsregime der technischen Normung und der Arzneimittelzulassung zeigen, dass es durch die Einführung funktional differenzierter Regulierungsverfahren in Verbindung mit der Verrechtlichung des Entscheidungsprozesses gelingen kann, alle beteiligten Akteure so auf allseits verbindliche Entscheidungskriterien zu verptlichten und in verfahrensinduzierte Kontrollschleifen einzubinden, dass sie unterschiedliche Beiträge zum Regulierungsprozess liefern und trotzdem insgesamt zu einer gemeinsamen Suche nach problemadäquaten Lösungen veranlasst werden. Die politisch legitimierten Akteure der Gesetzgebungsebene, besonders der Rat und das Parlament, lenken den Regulierungsprozess, indem sie im Rahmen europäischer Richtlinien und Verordnungen verbindliche Entscheidungsvorgaben erlassen, die hinreichend konkret sind, um den verfügbaren Entscheidungsspielraum auf der nachgeordneten Ebene signifikant zu beschränken. Darüber hinaus errichten sie geeignete Verfahren, die versprechen, dass sich die fallspezifischen Regulierungsentscheidungen tatsächlich an diesen inhaltlichen Vorgaben orientieren. Dagegen verzichten sie darauf, im Einzelfall in den nachgeordneten Entscheidungsprozess zu intervenieren oder fallspezifische Entscheidungen vorwegzunehmen. Dadurch wird es erheblich schwieriger, im politischen Entscheidungsprozess situative Einzelinteressen wirksam zu vertreten. Die auf der nachgeordneten Entscheidungsebene dominierenden Expertengremien übernehmen die Funktion der fallspezifischen Detailentscheidung im Licht der verbindlichen materiellen Vorgaben und im Schatten einer Kontrolle durch jeweils nachgelagerte Akteure. Dadurch entsteht eine hochgradig institutionalisierte Entscheidungssituation, die sowohl für interessenorientiertes Schachern als auch für technokratische Exzesse wenig Spielraum lässt. Für die Funktionsfähigkeit solcher Regulierungsregime ist es dagegen nicht von grundsätzlicher Bedeutung, ob die eigentliche Entscheidungstätigkeit staatlichen oder nicht-staatlichen Experten übertragen wird.

Die aufgetretenen Schwierigkeiten im Bereich der Spielzeugrichtlinie und der Lebensmitrelregulierung zeigen jedoch, dass die im Neuen Ansatz vorgesehene Ebenendif- 
ferenzierung allein nicht ausreicht, um machtbasiertes "Schachern“ um Vorteile zu verhindern. Für die Normung von Spielzeug gelten formell dieselben Verfahren wie für den Maschinenbereich. Es liegt an den lückenhaften materiellen Vorgaben, dass die Spielzeugnormung in einigen Teilbereichen erhebliche Konflikte hervorruft. Regulierungsprobleme sind auf solche Gebiete beschränkt, für die sich die an der Ausarbeitung der Spielzeugrichtlinie beteiligten Akteure nicht auf hinreichend detaillierte Sicherheitsvorgaben verständigen konnten und wo Konflikte ungelöst auf die nachgeordnete Entscheidungsebene verlagert wurden. Für die Lebensmittelregulierung fehlten inhaltliche Vorgaben über dic Ausgestaltung der Detailregulierung nahezu vollständig, sodass die Mitgliedstaaten im Zusammenwirken mit der Kommission über ausgedehnte Ermessensspielräume verfügten, ohne verbindlichen Entscheidungsvorgaben unterworfen zu sein. Ohne konkrete Vorgaben verfügen die Akteure, die im Rahmen des nachgeordneten Entscheidungsprozesses tätig sind, über erweiterte Entscheidungsspielräume, die sie für machtbasiertes Verhandeln nutzen können. Dies führt entweder zu Entscheidungsblockaden oder zu sachlich nicht nachvollziehbaren Kompromissentscheidungen.

\section{Literatur}

Abraham. John/Lewis, Graham, 2000: Regulating Medicines in Europe. Competition, Expertise and Public Health. London.

Ansell, Christopher/Vogel, David, 2006: The Contested Grovernance of European Food Safety Regulation, in: Ansell, Christopher/Vogel, David. (Hrsg.), What's the Beef? The Contested Governance of European Food Safety. Manchester, 3-32.

Axelrod, Robert, 1984: The Evolution of Cooperation. New York.

Benz, Arthur, 2005: Governance im Mehrebenensystem, in: Schuppert, Gunnar F (Hrsg.), Governance-Forschung. Vergewisserung über Stand und Perspektiven. Baden-Baden, 95-120.

Berghaus, Hartwig/Langner, Dirk, 1998: Das CE-Zeichen. Richtlinientexte, Fundstellen der harmonisierten Normen, Zertifizierungsverfahren, Prüfstellen. Loseblattsammlung. München.

Börzel, Tanja, 2005: European Governance. Nicht neu, aber anders, in: Schuppert, Gunnar F. (Hrsg.), Governance-Forschung. Vergewisserung über Stand und Perspektiven. Baden-Baden, 72-94.

Brennan. Geoffrey/Buchanan, James M., 1985: The Reason of Rules. Constitutional Political Econony. Cambridge.

Broscheid, Andreas/leick, Jïrgen, 2005: Towards a European FDA? The Review of European Pharmaceuticals Authorization (Paper prepared for the 2005 Meeting of the European Union Studies Association, 21.3. - 2.4.2005 in Austin, Texas).

CMS Cameron McKenna/Andersen Consulting, 2000: Evaluation of the Operation of Community l'rocedures for the Authorisation of Medicinal Products, in: hup://pharmacos.eudra.org/F2/ home.heml, 5.2.2006.

Collatz. Brigitte, 1996: Die neuen curopäischen Zulassungsverfahren für Arzneimittel. Insbesondere Verfahren und Rechrsschutz des Antragstellers und Zulassungsinhabers bei Zulassungsentscheidungen. Aulendorf.

Dressel, Kerstin, 2002: BSE - The New Dimension of Uncertainty. The Cultural Politics of Science and Decision-Making. Berlin.

Eberlein, Burkard/Grande, Edgar, 2005: Beyond Delegation. Transnational Regulatory Regimes and the EU Regulatory State, in: Journal of European Public Policy 12, 89-112.

Elster, Jon, 1979: Ulysses and the Sirens: Studies in Rationality and Irrationality. Cambridge. 
Feick, Jürgen, 2000: Marktzugangsregulierung. Nationale Regulierung, internationale Harmonisierung und europäische Integration, in: Czada, Roland/Lïtz, Susanne (Hrsg.), Die politische Konstitution von Märkten. Opladen, 228-249.

Feick, Jürgen, 2002: Regulatory Europeanization, National Autonomy and Regulatory Effectiveness. Marketing Authorization for Pharmaceuticals. Max-Planck-Institut für Gesellschaftsforschung. Discussion Paper 02/6. Köln.

Gehring, Thomas/Krapohl, Sebastian/Kerler, Michael/Stefanova, Sachka, 2005: Rationalität durch Verfahren in der Europäischen Union. Europäische Arzneimittelzulassung und Normung technischer Güter. Baden-Baden.

Gehring, Thomas, 2005: Gesellschaftliche Rationalität durch die Differenzierung von Entscheidungsverfahren, in: Gehring, Thomas/Krapobl, Sebastian/Kerler, Michael/Stefanova, Sachka, Rationalität durch Verfahren in der Europäischen Union. Europäische Arzneimittelzulassung und Normung technischer Güter. Baden-Baden, 27-61.

Gehring, Thomas/Krapohl, Sebastian, 2007: Supranational Regulatory Agencies Between Independence and Control: The EMEA and the Authorisation of Pharmaceuticals in the European Single Market, in: Journal of European Public Policy 14, 208-226.

Glaeske, Gerd/Hart, Dieter/Merkel, Harald, 1988: Regulierung des europäischen Arzneimittelmarktes durch nationales und europäisches Zulassungs- und Nachmarktkontrollrecht, in: Reich, Norbert (Hrsg.), Die Europäisierung des Arzneimittelmarktes: Chancen und Risiken. BadenBaden, 13-23.

Héritier, Adrienne, 1996: The Accommodation of Diversity in European Policy Making and its Outcomes. Regulatory Policy as a Patchwork. EUI Working Paper SPS No. 96/2.

Héritier, Adrienne/Mingers, Susanne/Knill, Christoph/Becka, Martina, 1994: Die Veränderung von Staatlichkeit in Europa. Ein regulativer Wettbewerb: Deutschland, Großbritannien und Frankreich in der Europäischen Union. Opladen.

Jachtenfuchs, Markus/Kohler-Koch, Beate, 1996: Regieren im dynamischen Mehrebenensystem, in: Jachtenfuchs, Markus/Kohler-Koch, Beate (Hrsg.), Europäische Integration. Opladen, 15-44.

Jachtenfuchs, Markus/Kohler-Koch, Beate, 2004: Governance in der Europäischen Union, in: Benz, Arthur (Hrsg.), Governance. Regieren in komplexen Regelsystemen. Wiesbaden, 77-101.

Joerges, Christian/Falke, JosefMicklitz, Hans-Wolfgang/Brïggemeier, Gert, 1988: Dic Sicherheit von Konsumgütern und die Entwicklung der Europäischen Gemeinschaft. Baden-Baden.

Joerges, Christian/Neyer, Jïrgen, 1997: Transforming Strategic Interaction Into Deliberative Problem-Solving: European Comitology in the Foodstuffs Sector, in: Journal of European Public Policy 4, 1350-1763.

Kerler, Michael, 2005: Die Regulierung von Spielzeug in der Furopäischen Union, in: Gehring. Thomas/Krapohl, Sebastian/Kerler, Michael/Stefanova. Sachka, Rationalität durch Verfahren in der Europäischen Union. Europäische Arzneimittelzulassung und Normung technischer Güter. Baden-Baden, 311-334.

Krapohl, Sebastian, 2005: Die europäische Arzneimittelzulassung, in: Gehring, Thomas/Krapohl, Sebastian/Kerler, Michael/Stefanova, Sachka, 2005: Rationalitat durch Verfahren in der Europäischen Union. Furopäische Arzneimittelzulassung und Normung technischer Güter. BadenBaden, 81-196.

Krapohl, Sebastian/Zurek, Karolina, 2006: The Perils of Committee Governance. Intergovernmental Bargaining During the BSE Scandal in the European Union, in: European Integration Online Papers 10, http://papers.ssrn.com/sol3/papers.cfm?abstract_id=901444, 2. Mai 2007.

Krapohl, Sebastian, 2007: Thalidomide, BSE and the Single Market. A Historical-Institutionalist Approach to Regulatory Regimes in the European Union, in: European Journal of Political Research $46,25-46$.

Majone, Giandomenico, 1997: The New European Agencies: Regulation by Information, in: Journal of European Public Policy 4, 262-275.

Majone, Giandomenica, 2000: The Credibility Crisis of Community Regulation, in: Journal of Common Market Studies 38, 273-302.

Majone, Giandomenico, 2001: Two Logics of Delegation: Agency and Fiduciary Relations in EU Governance, in: European Union Politics 2, 103-122. 
Majone, Giandomenico, 2002: Delegation of Regulatory Powers in a Mixed Polity, in: European Law Journal 8, 319-339.

Marks, Gary/Hooghe, Liesbet/Blank, Kermit, 1996: European Integration From the 1980s: StateCentric Versus Multi-Level Governance, in: Journal of Common Market Studies 34, 341-378.

Mayntz, Renate, 1988: Funktionale Teilsysteme in der Theorie sozialer Differenzierung, in: Mayntz, Renate/Rosewitz, Bernd/Schimank, Uwe/Stichweh, Rurdolf(Hrsg.), Differenzierung und Verselbstständigung. Zur Entwicklung gesellschaftlicher Teilsysteme. Frankfurt a. M., 11-44.

Millstone, Erik/van Zwanenberg, Patrick, 2001: Politics of Expert Advice. Lessons from the Early History of the BSE Saga, in: Science and Public Policy 28, 99-112.

Moe, Terry, 1990: The Politics of Structural Choice. Toward a Theory of Public Bureaucracy, in: Williamson, Oliver E. (Hrsg.), Organization Theory. From Chester Barnard to the Present and Beyond. New York, 116-153.

Moravesik, Andreu, 1998: The Choice for Europe. Social Purpose and State Power from Messina to Maastricht. Ithaca.

Nentwich, Michael, 1995: Das Lebensmittelrecht der Europäischen Union: Entstehung, Rechtsprechung, Sekundärrecht, nationale Handlungsspielräume. Wien.

Pelkmans, Jaques, 1987: The New Approach to Technical Harmonization and Standardization, in: Journal of Common Market Studies 25, 249-269.

Permanand, Govin/Mossialos, Elias, 2005: Constitutional Asymmetry and Pharmaceutical PolicyMaking in the European Union, in: Journal of European Public Policy 12, 687-709.

Pollack, Mark A., 2003: The Engines of European Integration. Delegation, Agency, and Agenda Setting in the EU. Oxford.

Rawls, Johm, 1998: Fine Theorie der Gerechtigkeit. Frankfurt a. M.

Scharpf, Fritz W., 1997: Introduction: The Problem-Solving Capacity of Multi-Level Governance, in: Journal of Furopean Public Policy 4, 520-538.

Scharpf. Fritz W., 2003: Politische Optionen im vollendeten Binnenmarkt, in: Jachtenfuchs, Markus (Hrsg.), Europäische Integration. Leverkusen.

Schelling. Thomas, 1995: The Strategy of Conflict. Cambridge.

Shicble. Christoph, 2003: Produktsicherheitsgesetz und europäisches Gemeinschaftsrecht. BadenBaden.

Shapiro, Martin, 1992: The Giving-Reasons Requirement, in: University of Chicago Law Forum, 180-220.

Shapiro, Martin, 2001: The Institutionalization of European Administrative Space, in: Stone Sweet, Alec/Sandholtz, Wayne/Fligstein. Neil (Hrsg.), The Institutionalization of Europe. Oxford, 94112.

Stefanova, Sachka, 2005: Die Umsetzung der Maschinenrichtlinie, in: Gehring, Thomas/Krapohl, Sebastian/Kerler, Michatl/Stefanova, Sachka, Rationalität durch Verfahren in der Europäischen Union. Europäische Arzneimittelzulassung und Normung technischer Güter. Baden-Baden, $247-274$.

Voelakou: Helmut, 1996: Private Regierungen in der Techniksteuerung. Eine sozialwissenschaftliche Analyse der technischen Normung. Frankfurt a. M.

Logel, Darid, 1998: The Cilobalization of Pharmaceutical Regulation, in: Governance: An International Journal of Policy Administration 11, 1-22.

Vos. Fllem, 1999: Insritutional Frameworks of Community Health and Safety legislation: Committees, Agencies and Private Bodies. Oxford.

Westlake, Martin, 1997: Mad Cows and Englishmen. The Institutional Consequences of the BSF. Crisis, in: Journal of Common Market Studies 35. Annual Review, 11-36. 